\title{
The effect of anesthetic techniques on postoperative outcomes of open prostatectomy in the era of enhanced recovery after surgery
}

\author{
(1) Mehmet Anıl Süzer ${ }^{1}$, (1) Umut Kara², (1) Hasan Kamburoğlu³ , (1) Ceyda Çaparlar ${ }^{4}$, (1) Mehmet Özgür Özhan', \\ (D) Mehmet Burak Eşkin²
}

${ }^{1}$ Cankaya Hospital, Clinic of Anesthesiology and Reanimation, Ankara, Turkey

2University of Health Sciences Turkey, Gülhane Training and Research Hospital, Clinic of Anesthesiology and Reanimation, Ankara, Turkey

${ }^{3}$ Tuzla State Hospital, Clinic of Anesthesiology and Reanimation, Istanbul, Turkey

4University of Health Sciences Turkey, Yildirim Beyazit Training and Research Hospital, Clinic of Anesthesiology and Reanimation, Ankara, Turkey

\section{Date submitted:}

29.12.2020

Date accepted:

08.02.2021

Online publication date:

15.12.2021

\section{Corresponding Author:}

Umut Kara, M.D., University of Health Sciences Turkey, Gülhane Training and Research Hospital, Clinic of Anesthesiology and Reanimation, Ankara, Turkey drumutkara@gmail.com

ORCID:

orcid.org/0000-0001-5233-8255

Keywords: Prostatectomy, general anesthesia, spinal anesthesia, ERAS

\begin{abstract}
Aims: As the enhanced recovery after surgery (ERAS) concept gains popularity in the surgical practice, the anesthetic technique has a more important effect on the postoperative course, especially in aged population. The aim of this study was to compare general anesthesia (GA) with spinal anesthesia (SA) regarding the perioperative outcomes with respect to the ERAS protocols in open prostatectomy (OP) for benign prostate hyperplasia.

Methods: This retrospective study included patients between 40 and 90 years of age who underwent elective OP between 2014 and 2020. Data were collected from hospital's database, patient files, and anesthesia charts. The exclusion criteria were malignancy, lost to followup, and missing data. Primary outcome measures were perioperative variables. Secondary outcome measures were factors influencing hospital discharge time.

Results: Of 105 patients (age, mean \pm SD: 68.3 \pm 5.7 years) included in the study, 61 patients were administered in GA (group GA) and 44 in SA (group SA). As the primary outcome measures, when compared with group SA, deliberate hypotensive anesthesia was required in more patients [26 (42.6\%) vs. 15 (34.1\%); $p=0.027$ ] and transfusion rate was higher (6.5\% vs. 4.5\%; $\mathrm{p}=0.044)$ in group GA. Mean visual analogue scale score $(3.6 \pm 1.1$ vs. $2.8 \pm 0.4 ; p=0.040)$ and opioid consumption ( $32.8 \pm 4.4$ vs. $26.3 \pm 4.9 \mathrm{mg} ; \mathrm{p}=0.088$ ) were higher, and time to first rescue analgesic use was also shorter ( $1.1 \pm 0.9$ vs. $4.7 \pm 1.3$ hours; $p=0.011)$ in group $G A$ when compared with group SA.
\end{abstract}

Conclusions: This study showed that SA was superior to GA in maintaining hemodynamic stability, reducing blood loss, complications, postoperative analgesia requirement, and time to discharge, which are the main goals of ERAS protocols.

\section{Introduction}

As men age, benign prostate hyperplasia (BPH) becomes a common disease with a prevalence up to $60 \%$ until $9^{\text {th }}$ decade (1). Although transurethral techniques such as resection, vaporization, and holmium or thulium laser enucleation of the prostate are widely used surgical treatments for BPH, open prostatectomy (OP), in other words, "simple" prostatectomy continues to be a treatment option in patients with a large prostate greater than $>70 \mathrm{~mL}(2)$. Compared to the transurethral techniques, it has been reported that OP offers several advantages including lower re-operation rate, higher life quality and patient satisfaction, and better symptom relief in patients with large BPH $(3,4)$. OP is performed mainly under general or spinal anesthesia (SA). The anesthetic technique is determined

${ }^{\odot}$ Copyright 2021 by the University of Health Sciences Turkey, Gülhane Faculty of Medicine / Gülhane Medical Journal published by Galenos Publishing House. 
primarily considering the balance of the risk and benefit, because the patients are old and have several comorbidities.

After the introduction of the enhanced recovery after surgery (ERAS) protocols in daily practice, their benefits on the outcomes are well established in major urological cancer surgeries, which provide a safer perioperative course with early hospital discharge (5). ERAS protocols include preoperative counseling, maintaining hemodynamic stability, avoiding venous thromboembolism and fluid overload, early mobilization, early feeding, and effective pain relief. The anesthetic technique plays an important role in reducing surgical stress response, complications, and length of hospital stay in the postoperative period, especially in high-risk patients with older age (6). The decision-making for an anesthetic technique is a collaborative process that is shared by the patient, anesthesiologist, and surgeon (7). However, patient's anxiety and fear for anesthesia continue to be determinant in the decision despite the risks related to the technique (8).

Contrary to the urological cancer surgery, the role of the anesthetic technique in achieving of ERAS goals is not investigated in simple prostatectomy surgery. The aim of this current study was to evaluate the effect of the anesthetic techniques on the outcome of the OP with respect to the ERAS protocols in patients with $\mathrm{BPH}$.

The primary outcome measures were perioperative variables which included hemodynamic stability, blood loss, transfusion of blood products, postoperative pain scores, first analgesic requirement, rescue analgesic consumption, time to first oral intake, time to mobilization, and complications. The secondary outcome measures were to identify the length of hospital stay and the factors influencing hospital discharge time.

\section{Methods}

\section{Study Design}

This retrospective study was conducted in an university hospital and a private hospital. Data were collected from electronic medical records, patient files, and anesthesia charts to identify patients who underwent OP under general anesthesia (GA) or SA between 2014 and 2020. The patients with the American Society of Anesthesiologists physical status classification 1-3, aged between 40 and 90 years, who had BPH diagnosis and underwent elective surgery were included in the study. Patients with a history of prostate or bladder cancer, urgent surgery, lost to follow-up, and missing data were excluded. The study was approved by the hospital's ethic commitee (Gülhane Training and Research Hospital, date: 11/30/2020; protocol no: 2020/443).

\section{Data Collection}

Information about the patients were reviewed to record 1) demographic characteristics, 2) type of anesthetic technique
(GA or SA), 3) intraoperative variables including operating time, hemodynamic disturbances, blood loss, blood transfusion, time to discharge from PACU, adverse events or complications, 4) postoperative variables including pain scores, analgesic consumption, time to mobilization and to oral intake (hours), and discharge time (days). Complications were classified according to the surgery surgery and anesthesia. The study followed the strengthening the reporting of observational studies in epidemiology (STROBE) guidelines.

\section{Anesthetic Technique}

All patients were administered GA or SA. Also, a hypotensive agent (nitroglycerin) was intravenously infused to achieve a deliberate hypotension in combination with tranexamic acid to reduce blood loss during the surgery.

All patients were administered a multi-modal analgesic regimen. Pain intensity was recorded using the visual analogue scale (VAS) by the service nurses. All patients were administered pethidine as the rescue analgesic.

\section{Statistical Analysis}

Statistical Package for the Social Sciences (SPSS) program (version 21.0; IBM SPSS Inc, Chicago, IL) was used to analyze data. Continuous variables were expressed as mean \pm standard deviation or median (interquartile range). Dichotomous variables were presented as percentages (\%). Normality of data was tested using the Kolmogorov-Smirnov test. A t-test or MannWhitney $\mathrm{U}$ test was used to compare normally or non-normally distributed variables. Categorical variables were compared using the chi-square $\left(\mathrm{X}^{2}\right)$ and Fisher's exact test. $\mathrm{P}$ value of $<0.05$ was considered statistically significant.

\section{Results}

A total of 121 files were evaluated and 16 files were excluded due to missing data. The remaining 105 patients were divided into two groups according to the anesthetic technique, as group GA $(n=61)$ and group SA $(n=44)$. The demographic data are shown in Table 1. The mean age was 68.3 5.7 (54-91) years and the patients in group GA was younger than those in group SA (62.9 \pm 5.3 years vs. $74.4 \pm 4.1$ years; $p=0.033$ ). The mean body mass index was similar between groups. Co-morbidity rate was $71.4 \%$ in the study population and the rate of patients with co-morbidity was higher in group SA than in group GA $(79.5 \%$ vs $65.6 \%, p=0.021 p<0.05)$. The mean operative time was similar between two groups.

\section{Primary Outcome Measure}

A deliberate hypotensive anesthesia was required in more patients in group GA compared to group SA $(42.6 \%$ vs. $34.1 \%$, $p=0.027)$. The mean blood loss and blood transfusion rate were higher in group GA than to group SA $(345.9 \pm 33.0 \mathrm{~mL}$ vs. $290.9 \pm 18.5 \mathrm{~mL} ; p=0.034$ and $6.5 \%$ vs. $4.5 \% ; p=0.044$ ). Time to oral intake was longer in group GA $(5.5 \pm 0.2$ vs. $3.1 \pm 0.6$ hours; 
$\mathrm{p}=0.033)$ whereas time to mobilization was similar $(6.8 \pm 1.1$ vs. $6.7 \pm 0.9$ hours; $p=0.766)$. Mean VAS scores were higher (3.6 \pm 1.1 vs. $2.8 \pm 0.4 ; p=0.040$ ), time to first rescue analgesic was lower ( $1.1 \pm 0.9$ vs. $4.7 \pm 1.3$ hours; $p=0.011)$, and mean opioid consumption was higher in group GA $(32.8 \pm 4.4$ vs. $26.3 \pm 4.9$ $\mathrm{mg} ; \mathrm{p}=0.088$ ), especially in the first postoperative 6 hours. The complication rate was higher in group GA than group SA $(37.7 \%$ vs. $20.5 \%, p=0.021$ ). No fatal complication was recorded (Table 2).

\section{Secondary Outcome Measure}

The mean length of hospital stay was $2.2 \pm 0.2$ (2-7) days, which was longer in group GA than in group SA $(2.4 \pm 0.1$ vs. $1.9 \pm 0.5$ days; $p=0.026$ ). A total of 11 patients ( 7 in group $G A$ and 4 in group SA) were discharged later than the mean discharge time due to the following reasons: a) atelectasis requiring therapy in 4 patients ( 3 patients in group GA and 1 patient in group SA), b) hemodynamic disturbance and/or arrhythmia at the postoperative 2. day (2 patients; one patient in each group), c) delirium (3 patients; 2 in group GA and 1 in group SA), d) prolonged postoperative bleeding (one patient in group GA), and e) dysregulation of the blood glucose homeostasis (one patient in group SA) (Table 3).
Difficulty in airway management was observed in two patients in group GA. Direct laryngoscopy and endotracheal intubation failed in these patients. One patient was awakened and scheduled for a further surgery under SA. The other patient was intubated using an intubating laryngeal mask airway. SA was failed in two patients in group SA.

\section{Discussion}

The results of the study showed that SA offered a more stable and safer perioperative period compared with the GA in patients who underwent OP, which is consistent with the aims of ERAS protocols due to several reasons;

I. Hypotensive anesthesia was required less often, which indicated SA maintained deliberate hypotension more efficiently compared to the GA during the intraoperative period.

II. Blood loss and transfusion rate were lower

III. Postoperative pain relief was more effective, especially in the immediate postoperative period. Opioid consumption and opioid-related side effects were lower.

IV. Patients were discharged earlier.

\begin{tabular}{|c|c|c|c|}
\hline Parameter & General anesthesia $(n=61)$ & Spinal anesthesia $(n=44)$ & ${ }^{*} p$ \\
\hline Age (years) & $62.9 \pm 5.3$ & $74.4 \pm 4.1$ & 0.033 \\
\hline Co-morbidity (n, \%) & $40(65.6)$ & $35(79.5)$ & 0.021 \\
\hline Hypertension (n, \%) & $23(37.7)$ & $19(43.2)$ & 0.033 \\
\hline Pulmonary disease (n, \%) & $2(3.3)$ & $2(4.5)$ & 0.044 \\
\hline Mixt (n, \%) & $17(27.8)$ & $14(31.8)$ & 0.039 \\
\hline Body mass index $\left(\mathrm{kgm}^{-2}\right)$ & $26.1 \pm 1.8$ & $27.2 \pm 2.6$ & 0.900 \\
\hline Operative time (min.) & $130.1 \pm 8.4$ & $134.6 \pm 7.1$ & 0.780 \\
\hline
\end{tabular}

\begin{tabular}{|c|c|c|c|}
\hline Parameter & $\begin{array}{l}\text { General anesthesia } \\
(n=61)\end{array}$ & $\begin{array}{l}\text { Spinal anesthesia } \\
(n=44)\end{array}$ & $\mathbf{p}^{*}$ \\
\hline Estimated blood loss (mL) & $345.9 \pm 33.0$ & $290.9 \pm 18.5$ & 0.034 \\
\hline Transfusion of blood products $(n, \%)$ & $4(6.6)$ & $2(4.5)$ & 0.044 \\
\hline VAS score $(0-10)$ & $3.6 \pm 1.1$ & $2.8 \pm 0.4$ & 0.040 \\
\hline Time to first rescue analgesic $(\mathrm{h})$ & $1.1 \pm 0.9$ & $4.7 \pm 1.3$ & 0.011 \\
\hline Opioid consumption (mg) & $32.8 \pm 4.4$ & $26.3 \pm 4.9$ & 0.038 \\
\hline
\end{tabular}




\begin{tabular}{|c|c|c|c|}
\hline Parameter & General anesthesia $(n=61)$ & Spinal anesthesia $(n=44)$ & $\mathbf{p}^{*}$ \\
\hline Hospital discharge time (day) & $2.4 \pm 0.1$ & $1.9 \pm 0.5$ & 0.026 \\
\hline Complication rate $(\mathrm{n}, \%)$ & $23(37.7)$ & $9(20.5)$ & 0.021 \\
\hline Hypo/hypertension (n, \%) & $6(9.83)$ & $3(6.8)$ & 0.017 \\
\hline Arrhythmia requiring treatment $(\mathrm{n}, \%)$ & $1(1.6)$ & $1(2.3)$ & 0.388 \\
\hline Desaturation $\left(\mathrm{SpO}_{2}<90\right.$ in room air) $(\mathrm{n}, \%)$ & $4(6.6)$ & $1(2.3)$ & 0.018 \\
\hline Atelectasis (n, \%) & $3(4.92)$ & $1(2.3)$ & 0.018 \\
\hline Nausea and vomiting $(\mathrm{n}, \%)$ & $6(9.83)$ & $2(4.5)$ & 0.039 \\
\hline Delirium (n, \%) & $3(4.92)$ & $1(2.3)$ & 0.031 \\
\hline
\end{tabular}

These results are consistent with previous studies that compared regional anesthesia with GA in patients undergoing radical prostatectomy and reported that a more stable and safer perioperative period was achieved with regional anesthesia, which reduced the discharge time $(9,10)$.

This finding is indeed not surprising because the perioperative course in simple prostatectomies is expected to be less complicated compared to the radical prostatectomy, as the latter involves surgical removal of vas deferens, seminal vesicles and lymph nodes.

ERAS protocols, which aim mainly to improve patient care and reduce postoperative complications have been introduced for over 20 years in surgical procedures (5). Although ERAS concepts are applied more commonly to major urological surgery such as radical prostatectomy and cystectomy, it may also be reasonable to implement ERAS protocols in "simple" prostate surgery because the procedure includes elderly patients with comorbidities, has potential risks for major bleeding, transfusion requirements and complications $(5,6,9,10)$.

Another notable finding of the current study was that GA was performed to younger and healthier patients, which suggests that those patients tended to prefer GA, or those with an advanced age and less healthy patients were directed to SA by the anesthesiologist to reduce perioperative complications. A recent study has revealed that the choice of the anesthetic technique is greatly influenced by the patient's preference, especially in favor of GA (11). In another study, it was reported that $58 \%$ of younger patients preferred GA because they did not want to receive visual and auditory stimuli during the surgical procedure (12).

On the other hand, the results of the current study showed that the complications rate was higher and discharge time was longer in the GA group. This can be interpreted as a benefit of regional anesthesia in a population at this age undergoing surgery. It is well-known that regional anesthesia offers several advantages over GA including lower complication rate, better postoperative pain relief, and reduced hospital discharge time.
However, several studies have showed that a considerable number of elderly patients still preferred GA to regional anesthesia $(13,14)$.

The reasons for refusal of regional anesthesia in patients older than 60 years were reported as limited knowledge about regional anesthesia $(20.3 \%)$, fear of being awake during operation $(18.7 \%)$, needle pain $(7.7 \%)$, backache $(11.5 \%)$, and previous negative experience with regional anesthesia (8.8\%) (14). However, the most interesting finding of the present study was that the most common cause of refusal of regional anesthesia (38.5\%) was the surgeon's preference. This finding suggests that the surgeons have a major impact on patient's preference since they are the primary care providers, whereas the anesthesiologist does not communicate with the patients until the pre-anesthetic visit. Additionally, the reason of the refusal may be that the patients are not allowed to choose their anesthesia with the anesthesiologist, or the anesthesiologist may not be involved effectively in the decision-making process. The studies indicate that patient's satisfaction increases as they participate in the selection of the anesthetic method, but they may also need to be directed to more appropriate anesthetic options to reduce complications and to improve outcomes $(14,15)$.

This study has several limitations. First, the retrospective design of the study may have a potential risk for bias among study groups. Although time to mobilization, an important criteria in ERAS protocols, was found similar between the groups, it may not reflect the exact mobilization time because the patients were not allowed to mobilize within postoperative six hours by the surgeons.

\section{Conclusion}

In conclusion, this study showed that, SA was superior to the GA in maintaining hemodynamic stability, reducing blood loss, complications and providing better postoperative pain relief with earlier hospital discharge, in patients who underwent OP for $\mathrm{BPH}$, which are the main goals of ERAS protocols. 


\section{Ethics}

Ethics Committee Approval: Approval of the Local Research Ethics Committee of our tertiary hospital was obtained before initiating the study (University of Medical Sciences Turkey, Gülhane Training and Research Hospital, project no: 2020/43, date: 11.30.2020).

Informed Consent: Retrospective study.

Peer-review: Externally peer-reviewed.

\section{Authorship Contributions}

Surgical and Medical Practices: M.A.S., U.K., H.K., Concept: M.A.S., M.Ö.Ö., M.B.E., Design: M.A.S., U.K., H.K., Data Collection or Processing: C.Ç., H.K., U.K. Analysis or Interpretation: M.B.E., C.Ç., Literature Search: M.B.E., M.Ö.Ö., Writing: M.A.S., M.Ö.Ö., M.B.E.

Conflict of Interest: No conflict of interest was declared by the authors.

Financial Disclosure: The authors declared that this study received no financial support.

\section{References}

1. Lim KB. Epidemiology of clinical benign prostatic hyperplasia. Asian J Urol. 2017;4:148-151.

2. Zargooshi J. Open prostatectomy for benign prostate hyperplasia: short-term outcome in 3000 consecutive patients. Prostate Cancer Prostatic Dis. 2007;10:374-377.

3. Montesi L, Quaresima L, Tiroli M, et al. Improvement of lower urinary tract symptoms and sexual activity after open simple prostatectomy: prospective analysis of 50 cases. Arch Ital Urol Androl. 2014;86:353-355.

4. Milicevic S, Grubor P, Lucic N. The evaluation of impact of $\mathrm{BPH}$ surgical treatment with the open prostatectomy and transurethral resection of the prostate methods on the quality of life. Med Arh. 2011;65:274-277.

5. Cerantola Y, Valerio M, Persson B, et al. Guidelines for perioperative care after radical cystectomy for bladder cancer: Enhanced Recovery After Surgery (ERAS(®)) society recommendations. Clin Nutr. 2013;32:879-887.
6. Rodrigues Pessoa R, Urkmez A, Kukreja N, Baack Kukreja $\mathrm{J}$. Enhanced recovery after surgery review and urology applications in 2020. BJUI Compass. 2020;1:5-14.

7. Stubenrouch FE, Mus EMK, Lut JW, Hesselink EM, Ubbink DT. The current level of shared decision-making in anesthesiology: an exploratory study. BMC Anesthesiol. 2017; $17: 95$.

8. Hwang SM, Lee JJ, Jang JS, Gim GH, Kim MC, Lim SY. Patient preference and satisfaction with their involvement in the selection of an anesthetic method for surgery. J Korean Med Sci. 2014;29:287-291.

9. Salonia A, Crescenti A, Suardi N, et al. General versus spinal anesthesia in patients undergoing radical retropubic prostatectomy: results of a prospective, randomized study. Urology. 2004;64:95-100.

10. Kofler O, Prueckner S, Weninger E, et al. Anesthesia for Open Radical Retropubic Prostatectomy: A Comparison between Combined Spinal Epidural Anesthesia and Combined General Epidural Anesthesia. Prostate Cancer. 2019;2019:4921620.

11. Capdevila X, Aveline C, Delaunay L, et al. Factors Determining the Choice of Spinal Versus General Anesthesia in Patients Undergoing Ambulatory Surgery: Results of a Multicenter Observational Study. Adv Ther. 2020;37:527-540.

12. Adıyeke $E$, Adıyeke L. Factors affecting general or regional anesthesia preference in patients with elective surgery. Medical Science and Discovery. 2020;7:570-574.

13. Rhee WJ, Chung CJ, Lim YH, Lee KH, Lee SC. Factors in patient dissatisfaction and refusal regarding spinal anesthesia. Korean J Anesthesiol. 2010;59:260-264.

14. Salam AA, Afshan G. Patient refusal for regional anesthesia in elderly orthopedic population: A cross-sectional survey at a tertiary care hospital. J Anaesthesiol Clin Pharmacol. 2016;32:94-98.

15. Hwang SM, Lee JJ, Jang JS, Gim GH, Kim MC, Lim SY. Patient preference and satisfaction with their involvement in the selection of an anesthetic method for surgery. J Korean Med Sci. 2014;29:287-291. 